\title{
CONTINUOUS LATTICES AND WHITEHEADIAN THEORY OF SPACE
}

\begin{abstract}
In this paper a solution of Whitehead's problem is presented: Starting with a purely mereological system of regions a topological space is constructed such that the class of regions is isomorphic to the Boolean lattice of regular open sets of that space. This construction may be considered as a generalized completion in analogy to the well-known Dedekind completion of the rational numbers $\mathbb{Q}$ yielding the real numbers $\mathbb{R}$. The argument of the paper relies on the theories of continuous lattices and "pointless" topology.
\end{abstract}

\section{CONTENTS}

1. Introduction, p. 36

2. Basics of the Whiteheadian theory of space, p. 38

3. Open sets as Whiteheadian regions, p. 41

4. Regular open representations of Whiteheadian regions, p. 44

5. Concluding remarks, p. 51 


\section{Introduction}

The Whiteheadian account of space considers spatial regions as the fundamental spatial entities. Points, lines, and surfaces are constructed from regions. In this paper I want to show that space conceived as a set of points endowed with a topological structure may be constructed from a purely mereological system of regions alone. More precisely, I want to show the following: starting with a mereological system $\langle W, \ll\rangle$ defined as a set of mereological individuals (regions) endowed with a primitive notion of interior parthood $\ll$ one can construct a topological space $\langle\operatorname{pt}(W), \mathscr{O}(\operatorname{pt}(W)\rangle$ such that the elements of $W$ are faithfully represented by regular open subsets of the point set $\mathrm{pt}(W)$. In other words, $W$ is isomorphic to the set $\mathscr{O}^{\star}(p \mathrm{t}(W))$ of regular open subsets of $\mathrm{pt}(W)$. Moreover, the basic mereological relation $\ll$ is represented by a standard topological relation of interior parthood, to wit, $x \ll y$ iff the closure $\operatorname{cl}(\mathrm{r}(x))$ of $\mathrm{r}(x)$ is compact and $\mathrm{r}(x) \subseteq \mathrm{r}(y), \mathrm{r}(x)$ and $\mathrm{r}(y)$ being the representing point sets of the regions $x$ and $y$, respectively.

Although this result is simple to state, and the idea of the proof is natural, the details are probably a bit messy, at least for the taste of philosophers. Nevertheless I'd like to emphasize that the argumentation is nowhere ad hoc. Rather, the result may be considered as a by-product of some general well-established strains of mathematical thought. Completing the rational numbers $\mathbb{Q}$ to real numbers $\mathbb{R}$ by Dedekind cuts may be considered as an early ancestor of the step from $\langle W, \ll\rangle$ to $\mathscr{O}(\mathrm{pt}(W))$ which leads to a topological representation of Whiteheadian regions by regular open sets. Indeed, one may characterize the Whiteheadian account of space as a generalized Dedekind completion. The right framework for this account turns out to be the theory of continuous lattices (cf. Gierz et al. 1980, Banaschewski and Hofmann (eds.) 1981).

The outline of the paper is as follows: in section 2 the basics of a Whiteheadian theory of space are recalled. The Whiteheadian theory is characterized as a mereological theory based on the concept of parthood. In section 3 an easy solution for the basic problem of any Whiteheadian theory of space is offered, to wit, the construction of point space from spatial regions. For this purpose we rely on the apparatus of the so-called theory of "pointless" topol- 
ogy. The result is that the mereological system $W$ of Whiteheadian regions can be characterized as a complete Heyting algebra of a special sort. This implies that the Whiteheadian regions can be represented in a 1-1-fashion by the open sets of a topological space $\langle p t(W), \mathscr{O}(p t(W)\rangle, \operatorname{pt}(W)$ being the set points and $\mathscr{O}(\mathrm{pt}(W))$ being a topology on $\mathrm{pt}(W)$ defined as a special class of subsets of $p \mathrm{t}(W)$ satisfying the familiar axioms (see, for instance Davey and Priestley 1990, 10.1A, p. 210). Although this construction provides a solution of Whitehead's problem, this solution cannot be considered as optimal. Among the open sets of a topological space there are many sets we do not like to play the role of well-formed Whiteheadian spatial regions, e.g. sets with cracks and holes. Hence, a better solution of Whitehead's problem should get along with a smaller, better behaved class of sets. In other words, one should restrict the class of representing sets from $\mathscr{O}(\mathrm{pt}(W))$ to some appropriate subclass of $\mathscr{O}(\mathrm{pt}(W))$. A natural candidate is the class $\mathscr{O}^{\star}(\mathrm{pt}(W))$ of regular open sets of $\mathrm{pt}(W)$. In contrast to non-regular open sets, regular open sets do not have low-dimensional holes and cracks. Hence, a representation based on $\mathscr{O}^{\star}(\operatorname{pt}(W))$ would be better solution of Whithehead's task. Since $\mathscr{O}^{\star}(X)$ as a lattice does not uniquely determine the underlying topological space $X$, such an approach is more complicated than that based on open sets. Beyond $\mathscr{O}^{\star}(\operatorname{pt}(W))$ some further ingredient is needed. Most authors who have dealt with this problem have looked for some non-mereological structure to overcome this difficulty. Some have even maintained that only non-mereological concepts can help. A main aim of this paper is to show that this contention is false. As will be shown in the following, a purely mereological relation « of interior parthood suffices to do the necessary work. More precisely, an enriched complete Boolean algebra of regions suffices for the task of constructing a topological representation of Whiteheadian regions. This is carried out in section 4 making heavy use of concepts and theorems of the theory of continuous lattices (cf. Gierz et al. 1980, Banaschewski and Hoffmann (eds.) 1981). The result may be described as follows: starting with a purely mereological system $\langle W, \ll\rangle$ a continuous Heyting algebra $\operatorname{Id}_{\ll}(W)$ is constructed which is isomorphic to the lattice of open sets $\mathscr{O}\left(\mathrm{pt}\left(\operatorname{Id}_{\ll}(W)\right)\right)$ of a space $\left\langle p t\left(\operatorname{Id}_{\ll}(W)\right), \mathscr{O}\left(\operatorname{pt}\left(\operatorname{Id}_{\ll}(W)\right)\right)\right\rangle$ in such a way that $W$ is isomorphic to the lattice $\mathscr{O}^{\star}\left(\mathrm{pt}\left(\operatorname{Id}_{\ll}(W)\right)\right.$ of regular open sets of $\mathrm{pt}\left(\operatorname{Id}_{\ll}(W)\right)$. In order to locate the approach of this paper in the general landscape of the various accounts of Whiteheadian theory of space, in section 5 we conclude with a discussion of Roeper's recent lengthy paper (Roeper 1997) that provides the most thorough-going and complete Whiteheadian account of space presently available. 


\section{Basics of the Whiteheadian theory of space}

The basic assumptions of a Whiteheadian theory of space may be succinctly stated as follows (cf. Forrest 1996, 128):

\section{Basic assumptions of Whiteheadian theory of space:}

(1) Regions are the fundamental spatial entities.

(2) Regions have no parts other than regions, and are parts of nothing other than regions.

(3) All regions have the same dimension as space (except the empty region).

(4) Regions may be faithfully represented by sets of points.

These assumptions do not fully determine which point sets may represent Whiteheadian regions. This can be explicated as follows. Assume that space is conceived of as a topological space $\langle X, \mathscr{O}(X)\rangle, X$ being the set of spatial points and $\mathscr{O}(X)$ a topological structure. ${ }^{1}$ According to the set of minimal assumptions of Whiteheadian theory of space we could take as the class of representatives of Whiteheadian regions the class of open sets $\mathscr{O}(X)$, the class of regular open sets $\mathscr{O}^{\star}(X)$, or the class of regular closed sets $\mathscr{C}^{\star}(X)$, all of which consist of sets having the same dimension as the whole space $X$ (ignore the empty set). Investing some more wealth of invention, even other classes of representatives may be found, e.g. the class $\mathscr{O}_{\mathrm{L}}(X)$ of Lebesgue-regular open sets (cf. Mormann 1998). In any case, according to the spirit of Whitehead's account, the class of representative point sets should be as small as possible. Hence, one should not take the class of all subsets which satisfy the requirements of basic assumptions of Whiteheadian theory of space as the class of representatives of Whiteheadian regions. The aim of a Whiteheadian account of space is, ceteris paribus, to reconstruct space from a base of regions that is as parsimonious as possible. After these general remarks let us recall some notions of mereology and fix some terminology which are necessary for the following considerations.

Denote the class of Whiteheadian regions of space by $W$. Then, according to assumptions (1)-(3) we may assume that $W$ is a mereological system in the sense that for spatial regions a parthood relation is defined which satisfies the familiar mereological axioms: if the region $x$ is part of the region $y$ this is denoted by $x \leq y$. Parthood is assumed to be reflexive, antisymmetric and transitive:

\footnotetext{
1 This is a minimal assumption. Otherwise, we could not speak of dimension.
} 
Basic assumptions of the parthood relation: Let $W$ be the class of Whiteheadian regions. Then the parthood relation $\leq$ defined on $W$ is assumed to satisfy at least the following conditions:

$$
\begin{gathered}
x \leq x, \\
x \leq y \& y \leq x \Longrightarrow x=y, \\
x \leq y \& y \leq z \Longrightarrow x \leq z .
\end{gathered}
$$

The system $\langle W, \leq\rangle$ (often denoted simply by $W$ ) is called a mereological system of Whiteheadian regions. In the following it is assumed throughout that $\langle W, \leq\rangle$ has a maximal element, to be interpreted as the whole of space. Moreover, for technical reasons, the existence of an empty region 0 which is to be part of any other region. 0 is called the trivial part.

For $\langle W, \leq\rangle$ the other mereological concepts are defined as usual: the regions $x$ and $y$ overlap iff they have a non-trivial common part, $x$ and $y$ are said to be disjoint iff they do not overlap. The region $y$ is a fusion of the set of regions $\left\{x_{i}: i \in I\right\}$ iff all $x_{i}$ are parts of $y$ and no part of $y$ is disjoint from each of the $x_{i}(i \in I)$. Moreover, in this paper it is assumed that the axiom of unrestricted fusion (UF) holds for $W$. This axiom states that for any ensemble $\left\{x_{i}: i \in I\right\}$ of spatial regions a unique fusion $\bigsqcup_{i \in I} x_{i}$ exists which satisfies the following condition:

Axiom of Universal Fusion (UF): For any ensemble of regions $\left\{x_{i}\right.$ : $i \in I\}$ there is a unique region $\bigsqcup_{i \in I} x_{i}$ such that every region $x_{i}$ is part of $\bigsqcup_{i \in I} x_{i}$, and there is no part of $\bigsqcup_{i \in I} x_{i}$ that does not overlap with at least one of the $x_{i}$.

As is shown in Mormann 1998a (UF) amounts to the assumption that the mereological system of Whiteheadian regions is a complete lattice $\langle W, \leq\rangle$. More precisely, the fusion of regions $\bigsqcup_{i \in I} x_{i}$ is to be interpreted as the supremum $\sup _{i \in I} x_{i}$ of the set $\left\{x_{i}: i \in I\right\}$ in the sense of lattice theory. Similarly, the overlap of regions may be interpreted as the infimum. Actually, if one wants a faithful topological representation one needs stronger assumptions which flow from the structure of the representing domains: for open representations $W$ has to be a complete Heyting algebra, while for regular open representations $W$ has to be a complete Boolean algebra.

After having rehearsed the rudiments of standard mereology, let us now recall some basic notions of topology to be used in the following. First note that the general representational assumption (4) can be rendered precise in several ways. In this paper, topological notions will be used for this task. Let 
$\langle X, \mathscr{O}(X)\rangle$ be a topological space, and denote the powerset of $X$ by $\mathcal{P}(X)$. For $x \in \mathcal{P}(X)$ the closure and the interior of $x$ with respect to $\mathscr{O}(X)$ are denoted by $\operatorname{cl}(x)$ and $\operatorname{int}(x)$, respectively. A set $x$ is open iff $\operatorname{int}(x)=x$, i.e. $x \in \mathscr{O}(X), x$ is closed iff $\operatorname{cl}(x)=x$. A set is regular open iff $\operatorname{int}(\operatorname{cl}(x))=x$. The lattice of regular open sets of $X$ is denoted by $\mathscr{O}^{\star}(X)$.

A representation of $W$ is a mapping $\mathrm{r}: W \rightarrow \mathcal{P}(X)$ which maps regions $x$ onto point sets $\mathrm{r}(x) \in \mathcal{P}(X)$ such that the mereological relations are represented by corresponding set theoretical relations, and some other appropriate requirements of structure-preservation are satisfied. For instance, the mereological parthood relation is represented by set-theoretical inclusion:

$$
x \leq y \Rightarrow \mathrm{r}(x) \subseteq \mathrm{r}(y) .
$$

These structural assumptions do not uniquely determine the class of sets which are to represent Whiteheadian regions. Intuitively, it is clear that not just any contrived subset of $X$ should be allowed to represent a region. Rather, Whiteheadian regions should be represented by nice or well-formed subsets of $X$. Hence, a fully fledged Whiteheadian account of space should restrict the range of the representation $r: W \rightarrow \mathcal{P}(X)$ to a suitable subset $\mathscr{N}(X) \subseteq \mathcal{P}(X)$ such that the elements of $\mathscr{N}(X)$ are nice subsets of $X$. In other words, a nice representation is a mapping $\mathrm{r}: W \rightarrow \mathscr{N}(X)$. What is to be understood by this informal notion of niceness is, of course, a matter of dispute, at least to some extent. In this paper topological concepts are employed for the explication of "niceness". More precisely, I'd like to concentrate on the following two kinds of representations:

Definition 2.1. Let $r: W \rightarrow \mathcal{P}(X)$ be a representation of the mereological system $W$ of Whiteheadian spatial regions.

(i) $r$ is an open representation if it maps regions onto the open sets of $X$. An open representation is denoted by $\mathrm{r}: W \rightarrow \mathscr{O}(X)$.

(ii) $r$ is a regular open representation if it maps Whiteheadian regions onto the regular open sets of $J$. Hence, a regular open representation may be denoted by $\mathrm{r}: W \rightarrow \mathscr{O}^{\star}(X)$.

As will be shown in the next section, for open representations the fusion of regions is represented by the set-theoretical union of the regions fused, i.e., if the regions $x_{i}$ are represented by $\mathrm{r}\left(x_{i}\right) \in \mathscr{O}(X)$ the fusion $\bigsqcup_{i \in I} x_{i}$ is represented by $\bigcup_{i \in I} r\left(x_{i}\right)$. This seems to be pretty obvious, of course. One should note however, that the corresponding result does not hold for regular open representations $\mathrm{r}: W \rightarrow \mathscr{O}^{\star}(X)$ to be constructed in section 4. Rather, 
for a regular open representation the fusion $\bigsqcup_{i \in I} x_{i}$ is to be represented by $\operatorname{int}\left(\operatorname{cl}\left(\bigcup_{i \in I} \mathrm{r}\left(x_{i}\right)\right)\right) .^{2}$ The reason is that in $\mathscr{O}^{\star}(X)$ the lattice theoretical counterpart of fusion, namely the supremum is not the set theoretical union but rather $\operatorname{int}\left(\operatorname{cl}\left(\bigcup_{i \in I} \mathrm{r}\left(x_{i}\right)\right)\right)$.

\section{Open sets as Whiteheadian regions}

A straightforward solution of Whitehead's problem is available for those who are content with representing the class $W$ of Whiteheadian regions by the class $\mathscr{O}(\mathrm{pt}(W))$ of open sets of an appropriate topological space $\langle\operatorname{pt}(W), \mathscr{O}(\operatorname{pt}(W)\rangle$. As is well-known, the lattice $\mathscr{O}(X)$ of open sets of any topological space $\langle X, \mathscr{O}(X)\rangle$ is a Heyting-algebra. Under mild restrictions, the underlying set of points $X$ can be reconstructed from $\mathscr{O}(X)$ by the standard spectral construction. ${ }^{3}$ For this purpose we have to recall some concepts and results of the theory of "pointless topology" (cf. Johnstone 1983, Mac Lane, Moerdijk 1992). Pointless topology has shown that the dependence on points in topology is not as deep as one might have expected. Large parts of topology may be done, at least in principle, without recourse to points. ${ }^{4}$ For many issues it suffices to deal with the lattice $\mathscr{O}(X)$ of open sets, ignoring the underlying set $X$. It is even possible, to reconstruct the set $X$ from the lattice $\mathscr{O}(X)$. The recipe for this construction is practically the same as the one Stone employed in the proof of his famous representation theorem (cf. Stone 1936). One topologizes the set of prime ideals $\mathrm{pt}(W)$ of $W$ in an appropriate way thereby getting a topological space $\langle\operatorname{pt}(W), \mathscr{O}(\operatorname{pt}(W))\rangle$. Under mild restrictions, $\mathscr{O}(\mathrm{pt}(W))$ is isomorphic to $W$. This yields the desired topological representation of $W$. The details are as follows:

Definition 3.1. Let $L$ be a complete lattice. Denote the 2-point set $\{0,1\}$ by 2 . Then 2 may be considered as a complete lattice, taking 0 as the bottom and 1 as the top element and the obvious lattice theoretical operations.

(i) A map $a: L \rightarrow 2$ preserves infinite joins iff for all $K \subseteq L$ the following holds $a(\bigvee K)=\bigvee\{a(k): k \in K\}$; the map $a$ preserves finite meets iff $a(x \wedge y)=a(x) \wedge a(y)$, for all $x, y \in L$. Denote by $p t(L)$ the set of mappings

\footnotetext{
2 These problems are discussed in some detail in Mormann 1998. See also Forrest 1996.

3 These restrictions are met in the case of Euclidean space for which the Whiteheadian account of space has been originally designed for.

${ }^{4}$ In terms of category theory this may be rendered precise as follows: the (lattice-theoretical) category of spatial locales is equivalent to the (topological) category of sober spaces (cf. Mac Lane, Moerdijk 1992, 479).
} 
$a: L \rightarrow 2$ which preserve infinite joins and finite meets. The set $\operatorname{pt}(L)$ is called the set of points of $L$.

(ii) $L$ is said to have enough points iff the points of $L$ separate the elements of $L$, i.e., for any two elements $x, y \in L$ there is an $a \in \operatorname{pt}(L)$ such that $a(x) \neq a(y)$.

The rationale of Definition 3.1 is the following: suppose $L$ is the lattice $\mathscr{O}(X)$ of open sets of some topological space $\langle X, \mathscr{O}(X)\rangle$. If $\mathscr{O}(X)$ is well-behaved, there is a natural bijection $\mathrm{r}: X \rightarrow \mathrm{pt}(\mathscr{O}(X))$ defined by $\mathrm{r}(\alpha)(x)=1$, if $\alpha \in x$, and $\mathrm{r}(\alpha)(x)=0$, otherwise. Hence, an "ordinary" point $\alpha$ defines a point in the sense of Definition 3.1. Hence, one may call the maps $a: \mathscr{O}(X) \rightarrow 2$ points of $X$, or even points of $\mathscr{O}(X)$. The point of this definition of points of $\mathscr{O}(X)$ is that it only depends on the lattice properties of $\mathscr{O}(X)$ and not on $X$ itself.

Definition and Lemma 3.2. (Mac Lane and Moerdijk 1991, IX, Definition 2, Proposition 2) Let $\langle X, \mathscr{O}(X)\rangle$ be a topological space.

(i) The topological space $X$ is sober iff for $p \in \mathscr{O}(X)$ such that

$$
\begin{gathered}
p \neq X, \\
x \cap y \subseteq p \Longrightarrow x \subseteq p \text { or } y \subseteq p \text { for all } x, y \in \mathscr{O}(X)
\end{gathered}
$$

there is a unique point $\alpha \in X$ with $p=X-\operatorname{cl}(\{\alpha\})$.

(ii) The mapping $\mathrm{r}: X \rightarrow \operatorname{pt}(\mathscr{O}(X))$ is a bijection iff $X$ is sober.

Sober spaces abound, for instance, Hausdorff spaces are sober (but not all sober spaces are Hausdorff). Hence, there is a large supply of spaces for which the representation $\mathrm{r}: X \rightarrow \operatorname{pt}(\mathscr{O}(X))$ is a bijection. For $x \in \mathscr{O}(X)$ define $\mathrm{r}(x)$ as $\{a: a \in \operatorname{pt}(\mathscr{O}(X)) \& a(x)=1\}$. Hence, the map $\mathrm{r}$ induces a map r: $\mathscr{O}(X) \rightarrow \mathcal{P}(\operatorname{pt}(\mathscr{O}(X)))$. As is easily seen, $\{\mathrm{r}(x): x \in \mathscr{O}(X)\}$ defines a topology $\mathscr{O}(\operatorname{pt}(\mathscr{O}(X)))$ on $\operatorname{pt}(\mathscr{O}(X))$. If $\langle X, \mathscr{O}(X)\rangle$ is sober $\mathrm{r}: X \rightarrow$ $\operatorname{pt}(\mathscr{O}(X))$ is a homeomorphism with respect to the topologies $\mathscr{O}(X)$ and $\mathscr{O}(\operatorname{pt}(\mathscr{O}(X)))$.

The definition of $\operatorname{pt}(\mathscr{O}(X))$ does not depend on the fact that the elements of $\mathscr{O}(X)$ are point sets. The only feature of $\mathscr{O}(X)$ employed in the construction of $\operatorname{pt}(\mathscr{O}(X))$ is that $\mathscr{O}(X)$ is a complete lattice. More precisely, the elements of $\operatorname{pt}(\mathscr{O}(X))$ are just the prime elements of $\mathscr{O}(X)$ (cf. Mac Lane and Moerdijk 1991, p. 474). Hence, this construction may be carried out for any (complete) lattice $L$. Consequently, the elements of $p t(L)$ are called the 
points of $L$ although the elements of $L$ may not have been defined as point sets at all. The set $\operatorname{pt}(L)$ provides the basis for a topological representation of $L$ which may be defined as follows:

Definition and Lemma 3.3. Let $L$ be a complete lattice, and $p \mathrm{t}(L)$ the set of points of $L$. Define the mapping $\mathrm{r}: L \rightarrow \mathcal{P}(\operatorname{pt}(L))$ by $\mathrm{r}(x):=\{a:$ $a(x)=1\}$. The sets $\{r(x): x \in L\}$ define a topology $\mathscr{O}(\operatorname{pt}(L))$ on $\operatorname{pt}(L)$. Hence, $r$ may be conceptualized as a map $\mathrm{r}: L \rightarrow \mathscr{O}(\mathrm{pt}(L))$, and $\mathrm{r}$ may be called the topological representation of $L$.

Proof. One has to show that the class of sets $\{\mathrm{r}(x): x \in L\} \subseteq \mathcal{P}(\operatorname{pt}(L))$ is closed with respect to arbitrary union and finite intersection. This immediately follows from the fact that the elements of $\mathrm{pt}(L)$ preserve arbitrary joins and finite meets.

Corollary 3.4. (Topological representation of Whiteheadian mereological systems)

(i) If $W$ is a system of Whiteheadian regions for which the axiom (UF) of universal fusion holds then $W$ may be conceived of as a complete lattice $\langle W, \leq\rangle$. Then there is a canonical representation $r: W \rightarrow \mathscr{O}(\operatorname{pt}(W))$. The topology $\mathscr{O}(\operatorname{pt}(W))$ on $\mathrm{pt}(W)$ is defined by $\mathscr{O}(\mathrm{pt}(W)):=\{\mathrm{r}(x): x \in W\}$.

(ii) If $W$ is a complete Heyting algebra with enough points in the sense of Definition 3.1 the representation $\mathrm{r}: W \rightarrow \mathscr{O}(\mathrm{pt}(W))$ is a lattice isomorphism and $\mathscr{O}(\mathrm{pt}(W))$ is sober.

The Corollary 3.4 may be called the easy solution of the basic task of a Whiteheadian theory of space: given a mereological system $\langle W, \leq\rangle$ of Whiteheadian regions having the structure of a complete Heyting algebra with enough points, a topological space $\langle\operatorname{pt}(W), \mathscr{O}(\operatorname{pt}(W)\rangle$ is constructed which faithfully represents $W$ in the sense that $W$ and $\mathscr{O}(\mathrm{pt}(W))$ are isomorphic as Heyting algebras. As has already been explained in the introduction this solution of Whitehead's problem leaves something to desire inofar as the lattice $\mathscr{O}(p t(W))$ of open sets is rather large and contains elements one would intuitively not consider as well-formed Whiteheadian regions. Hence, one should attempt to restrict the class of region-representing point sets from $\mathscr{O}(\mathrm{pt}(W))$ to a smaller, better behaved subclass of $\mathscr{O}(\mathrm{pt}(W))$. In the next section this is achieved by replacing the lattice $\mathscr{O}(\operatorname{pt}(W))$ by a lattice $\mathscr{O}\left(\operatorname{pt}\left(\operatorname{Id}_{\ll}(W)\right)\right)$ such that $W$ is isomorphic to the lattice $\mathscr{O}^{\star}\left(\operatorname{pt}\left(\operatorname{Id}_{\ll}(W)\right)\right)$ of regular open sets of the topological space $\left\langle\operatorname{pt}\left(\operatorname{Id}_{\ll}(W)\right), \mathscr{O}\left(\operatorname{pt}\left(\operatorname{Id}_{\ll}(W)\right)\right)\right\rangle$. Moreover it will be shown that $\operatorname{Id}_{\ll}(W)$ and $\mathscr{O}\left(\operatorname{pt}\left(\operatorname{Id}_{\ll}(W)\right)\right)$ are isomorphic. 


\section{Regular open representations of Whiteheadian regions}

Now we come to the central topic of this paper, namely, the construction of a topological space $\langle X, \mathscr{O}(X)\rangle$ for a mereological system $W$ of Whiteheadian regions such that they are represented by regular open sets $\mathscr{O}^{\star}(X)$. Since $\mathscr{O}^{\star}(X)$ is a complete Boolean algebra this amounts to the assumption that $\langle W, \leq\rangle$ is a complete Boolean algebra. Then it will be shown that the mereological system $W$ may be faithfully represented by the complete Boolean algebra $\mathscr{O}^{\star}(X)$ of the regular open subsets of $X$. Before we delve into the technical details I want to outline the construction in general terms. The construction process is carried out in six stages:

(1) We start with a mereological system $\langle W, \ll\rangle$ consisting of a set of Whiteheadian regions endowed with the relation $\ll$ of interior parthood. ${ }^{5} \mathrm{In}$ this stage, the only assumption on $\ll$ is transitivity.

(2) The relation $\ll$ is used to defined a partial order $\leq$ on $W$. Concerning the relation $\leq$, two groups of assumptions are made: (a) The relational system $\langle W, \leq\rangle$ is assumed to be a complete Boolean algebra; (b) the relations $\ll$ and $\leq$ are related in a specific way which renders $\ll$ an auxiliary relation of $\leq$ (cf. Gierz et al. 1980). Thereby one gets an enriched Boolean algebra $\langle W, \ll, \leq\rangle$.

(3) From $\langle W, \ll, \leq\rangle$ the lattice $\operatorname{Id}_{\ll}(W, \leq)$ of Dedekind ideals is constructed. It is well-known that $\operatorname{Id}_{\ll}(W)$ is a continuous Heyting-algebra.

(4) Since $\operatorname{Id}_{\ll}(W)$ is a continuous Heyting algebra one can prove that it is isomorphic to the Heyting algebra $\mathscr{O}\left(p t\left(\operatorname{Id}_{\ll}(W)\right)\right)$ of open sets of a locally compact Hausdorff space $\left\langle p t\left(\operatorname{Id}_{\ll}(W), \mathscr{O}\left(p t\left(\operatorname{Id}_{\ll}(W)\right\rangle\right.\right.\right.$.

(5) There is an embedding of $r: W \rightarrow \operatorname{Id}_{\ll}(W)$ such that $W$ may be considered in a natural way as a subset of $\operatorname{Id}_{\ll}(W)$. Moreover, one can prove that $W$ is isomorphic to the algebra $\mathscr{O}^{\star}\left(\operatorname{pt}\left(\operatorname{Id}_{\ll}(W)\right)\right)$ of regular open sets of $\mathrm{pt}\left(\operatorname{Id}_{\ll}(W)\right)$.

(6) If $\langle X, \mathscr{O}(X)\rangle$ is a locally compact Hausdorff space the complete Boolean algebra $\mathscr{O}^{\star}(X)$ may be endowed with the standard auxiliary relation $\ll_{\mathscr{O}(X)}$ yielding an enriched lattice $\left\langle\mathscr{O}^{\star}(X), \leq\right.$, « such that the continuous lattices $\operatorname{Id}_{\ll}\left(\mathscr{O}^{\star}(X)\right)$ and $\mathscr{O}(X)$ are isomorphic.

In order to get an intuitive idea about what is going on during the several stages it may be expedient to look at the construction process from

\footnotetext{
${ }^{5}$ Beware: the notion « of interior parthood is different from those defined in Simons 1987 or Roeper 1997.
} 
hindsight, i.e., after a representing space $\langle X, \mathscr{O}(X)\rangle$ for $\mathscr{O}^{\star}(X)(=W)$ has already been constructed. Hence, assume $\langle X, \mathscr{O}(X)\rangle$ to be a locally compact Hausdorff space and $\mathscr{O}^{\star}(X)$ its complete Boolean algebra of regular open sets. Assume that the system $W$ of Whiteheadian regions has been identified with the lattice $\mathscr{O}^{\star}(X)$. On the lattice $\mathscr{O}^{\star}(X)$ an auxiliary relation $\ll_{\mathscr{O}}(X)$ is defined by

$$
x \ll_{\mathscr{O}(X)} y \stackrel{\mathrm{df}}{\Longleftrightarrow} \operatorname{cl}(x) \text { is compact and } \operatorname{cl}(x) \subseteq y .
$$

This relation is, according to all intuitive demands, a good relation of interior parthood. For $\ll_{\mathscr{O}(X)}$ we may define a relation $\leq$ by

$$
x \leq y \stackrel{\mathrm{df}}{\Longleftrightarrow} \forall z\left(z \ll_{\mathscr{O}(X)} x \Rightarrow z \ll_{\mathscr{O}(X)} y\right)
$$

Since $\langle X, \mathscr{O}(X)\rangle$ is a regular space, the relation $\leq$ can be proven to be just the ordinary set theoretical inclusion:

$$
x \leq y \Longleftrightarrow x \subseteq y .
$$

Hence, $\left\langle\mathscr{O}^{\star}(X), \leq\right\rangle$ is a complete Boolean algebra. Moreover, since $\langle X, \mathscr{O}(X)\rangle$ is a locally compact Hausdorff space all the assumptions 4.3 on the relations between $\ll_{\mathscr{O}(X)}$ and $\leq$ are seen to be satisfied. This renders $\left\langle\mathscr{O}^{\star}(X), \ll, \leq\right\rangle$ an enriched Boolean algebra. Then, one may define the lattice $\operatorname{Id}_{\ll}\left(\mathscr{O}^{\star}(X)\right)$ of Dedekind ideals of $\mathscr{O}^{\star}(X)$ and eventually prove that $\operatorname{Id}_{\ll}\left(\mathscr{O}^{\star}(X)\right)$ is isomorphic to $\mathscr{O}(X)$ in such a way that the subset $\mathscr{O}^{\star}(X)$ of $\operatorname{Id}_{\ll}\left(\mathscr{O}^{\star}(X)\right)$ is isomorphic to the fixpoint set of the double negation operator $\neg \neg$ of the Heyting algebra $\operatorname{Id}_{\ll}\left(\mathscr{O}^{\star}(X)\right)$.

After this sketch of the general strategy, let us start with the technical details.

Stage 1. Let $W$ be the set of Whiteheadian regions endowed with the primitive notion of "interior parthood" to be denoted by «. Hence, for mereological individuals $x$ and $y$ the relation $x \ll y$ may be read as " $x$ is an interior part of y". It should be noted, however, that $\ll$ is not restricted to mereology. Following (Gierz et al. 1980) the relation $\ll$ is sometimes called "the way below relation" (cf. ibidem, p. 38ff). In this stage, the relation « is only assumed to be transitive:

$$
x \ll y \ll z \Longrightarrow x \ll z .
$$

It should be noted that $\ll$ is not assumed to be reflexive. That is to say, usually, an individual is not an interior part of itself. The set of mereological 
individuals $W$ endowed with the relation $\ll$ is denoted by $\langle W, \ll\rangle$. The mereological system $\langle W, \ll\rangle$ provides the basis for the following steps. One should further note that at this stage $W$ is not assumed to be endowed with a relation of "ordinary" parthood. This will be defined in the next stage.

Stage 2. The second stage of the construction consists in defining a concept of "parthood" and giving some axioms of $\leq$ and its relation to the primitive notion of interior parthood $\ll$.

Definition 4.1. Let $\langle W, \ll\rangle$ be a mereological system of Whiteheadian regions endowed with a transitive relation $\ll$ of interior parthood. Then the relation $\leq$ of parthood is defined as follows:

$$
x \leq y \stackrel{\mathrm{df}}{\Longleftrightarrow} \forall z(z \ll x \Rightarrow z \ll y) .
$$

Due to the transitivity of the relation of interior parthood, $x \ll y$ implies $x \leq y$. The reverse relation does (and should) not hold, however. Rather, the relation between the relations $\ll$ and $\leq$ is pretty complex. It is captured by the following two classes of assumptions:

Basic assumptions I 4.2. The structure $\langle W, \leq\rangle$ is a complete Boolean algebra. The maximal and the minimal element of this algebra is denoted by 1 and 0 , respectively. As usual, the operations of sup and inf (with respect to $\leq$ ) are denoted by $\vee$ and $\wedge$, respectively. The Boolean complement of $x$ is denoted by $x^{\star}$.

Basic assumptions II 4.3. For all $x, y, z, u \in W$ the following relations hold:

(i) $u \leq x \ll y \leq z$ implies $u \ll z$.

(ii) $x \ll z$ and $y \ll z$ together imply $x \vee y \ll z$.

(iii) $0 \ll x$.

(iv) $x \ll z$ and $x \neq z$ together imply $x \ll y \ll z$ for some $y$ and $x \neq y$ (strong interpolation property).

(v) $x \ll y$ and $x \ll z$ implies $x \ll y \wedge z$.

Generally, let $\langle L, \leq\rangle$ be any lattice, and $\ll$ a further relation defined on $L$. The structure $\langle L, \ll, \leq\rangle$ is called an enriched lattice iff $4.3(\mathrm{i})-(\mathrm{v})$ hold. The relation $\ll$ is called an auxiliary relation. It should be noted that auxiliary relations are not uniquely determined, i.e., for a lattice $\langle L, \leq\rangle$ there may 
be many different auxiliary relations $\ll^{\prime}$. Hence, one and the same lattice $\langle L, \leq\rangle$ may give rise to various enriched lattices $\left\langle L, \ll^{\prime}, \leq\right\rangle$. For instance, the relation $\leq$ renders $\langle L, \leq, \leq\rangle$ an enriched lattice. This finishes the second stage of the construction process.

Stage 3. All the necessary ingredients for the definition of the lattice $\operatorname{Id}_{\ll}(W)$ of Dedekind ideals are now available. First recall the definition of an ordinary ideal of a lattice $L$ : a subset $I \subseteq L$ is an ideal of $L$ iff $I=\{y: y \leq x$ for some $x \in I\}$, and for $a, b \in I, a \vee b \in I$. The class of all ideals of $L$ is denoted by $\operatorname{Id}(L)$ and called the ideal completion of $L$ (cf. Vickers 1989, Definition 9.1.1). Then we may define:

Definition 4.4. Let $\langle L, \leq, \ll\rangle$ be an enriched lattice. An ideal $I \in \operatorname{Id}(L)$ is a called a Dedekind ideal (with respect to the auxiliary relation $\ll$ ) if whenever, $a \in I$, then $a \ll b$ for some $b \in I$. The set of Dedekind ideals is denoted by $\operatorname{Id}_{\ll}(L)$.

The sets $\operatorname{Id}(L)$ and $\operatorname{Id}_{\ll}(L)$ are naturally ordered by set theoretical inclusion. Hence, one may ask if they may be lattices of some specific kind. With respect to this question we start with the following result:

Proposition 4.5. (cf. Gierz et al. 1980, chapter I, Lemma 2.10, and Gierz and Keimel 1981, 2.6) If $\langle L, \leq, \ll\rangle$ is an enriched lattice the set of Dedekind ideals $\operatorname{Id}_{\ll}(L)$ is a complete lattice.

Actually, depending on the structure of $\langle L, \leq\rangle$, much stronger assertions may be proved. To state them, we need the following definition which introduces for all complete lattices a standard auxiliary relation:

Definition and Lemma 4.6. Let $\langle L, \leq\rangle$ be a complete lattice.

(i) The standard auxiliary relation $\ll_{L}$ is defined by:

$x \ll_{L} y \stackrel{\mathrm{df}}{\Longleftrightarrow}$ for all directed subsets $D \subseteq L$ the relation $y \leq \sup D$ implies the existence of a $d \in D$ with $x \leq d$.

AS is easily seen, $\left\langle L, \ll_{L}, \leq\right\rangle$ is an enriched lattice.

(ii) $L$ is said to be a continuous lattice iff $\ll_{L}$ satisfies the axiom of approximation:

$$
x=\sup \left\{u \in L: u \ll_{L} x\right\} .
$$


If (A) holds, every element of $L$ can be approximated by elements which are its interior parts. This amounts to the fact that the relation $\leq$ may be defined by $\ll_{L}$ via Definition 4.1. More precisely, one defines a relation $\leq_{L}$ by

$$
x \leq_{L} y \stackrel{\mathrm{df}}{\Longleftrightarrow} \forall z(z \ll x \Rightarrow z \ll y) .
$$

Then one shows that $x \leq y$ iff $x \leq_{L} y$. Hence, we can think of continuous lattices $\langle L, \leq\rangle$ as structures which arise from structures $\langle L, \ll\rangle$ for which an appropriate relation $\leq_{\langle L, \ll\rangle}$ can be defined which satisfies the above mentioned requirements 4.3 and $4.6(\mathrm{ii})$.

Example. Let $X$ be a locally compact Hausdorff space. Then the lattice $\mathscr{O}(X)$ is a continuous lattice (cf. Gierz et. al 1980, I, 1.7, p. 42). In topological terms the standard auxiliary relation $\ll_{\mathscr{O}(X)}$ may be expressed as follows (ibidem, I, 1.4., Proposition, p. 40-41):

$$
x \ll_{\mathscr{O}(X)} y \Longleftrightarrow \operatorname{cl}(x) \subseteq y \text { and } \operatorname{cl}(x) \text { is compact. }
$$

As is exemplified by the following proposition continuous lattice provide the appropriate framework for the regular Whiteheadian account of space.

Proposition 4.7. (cf. Gierz and Keimel 1981, 3.2, 5.7) If $\langle W, \leq, \ll\rangle$ is an enriched complete Boolean algebra the complete lattice $\operatorname{Id}_{\ll}(W)$ of Dedekind ideals is a continuous Heyting algebra.

A proof of Proposition 4.7 may be sketched as follows: First note that $\operatorname{Id}(W)$ is a complete Heyting algebra. Due to $4.3(\mathrm{v})$ one can prove that $\operatorname{Id}_{\ll}(W)$ is a sublattice of $\operatorname{Id}(W)$ (cf. Gierz and Keimel 1981, 5.4). Since $\operatorname{Id}_{\ll}(W)$ is also a complete lattice it is a complete Heyting algebra too. Moreover, due to (Gierz et. al. 1980, I, Lemma 2.10) the Dedekind completion $\operatorname{Id}_{\ll}(W)$ of $W$ is a continuous Heyting algebra.

This finishes stage 3 . In the following stages it is shown that the lattice of Dedekind ideals $\operatorname{Id}_{\ll}(W)$ gives rise to a topological space whose lattice of regular open sets is isomorphic to $\langle W, \leq\rangle$. The details are as follows:

StAGe 4. $W$ may be embedded into $\operatorname{Id}_{\ll}(W)$ by the natural map $\mathrm{r}: W \rightarrow$ $\operatorname{Id}_{\ll}(W)$ defined by $\mathrm{r}(x):=\{y: y \ll x\}$. Then the following holds (cf. Gierz et al. 1980, III, 4.20):

$$
x \ll y \Longleftrightarrow \mathrm{r}(x) \ll_{\mathrm{Id}_{\ll}(W)} \mathrm{r}(y) .
$$


Moreover, $\operatorname{Id}_{\ll}(W)$ being a continuous Heyting algebra implies that it has enough points in the sense of Definition 3.1. Hence, it is isomorphic to the Heyting algebra of open sets of some topological space $\left\langle p t\left(I d_{\ll}(W)\right)\right.$, $\left.\mathscr{O}\left(\operatorname{pt}\left(\operatorname{Id}_{\ll}(W)\right)\right)\right\rangle$ (cf. Gierz et al. 1980, 5.16). More precisely, the following holds:

Theorem 4.8. Let $\langle W, \ll, \leq\rangle$ be an enriched complete Boolean algebra. Then there is a locally compact Hausdorff space $\left\langle p t\left(\operatorname{Id}_{\ll}(W)\right)\right.$, $\left.\mathscr{O}\left(\operatorname{pt}\left(\operatorname{Id}_{\ll}(W)\right)\right)\right\rangle$ such that $\mathscr{O}\left(\operatorname{pt}\left(\operatorname{Id}_{\ll}(W)\right)\right)$ and $\operatorname{Id}_{\ll}(W)$ are isomorphic Heyting algebras.

This clinches the fourth stage of the construction. Next it is shown that $W$, via the embedding $i: W \rightarrow \operatorname{Id}_{\ll}(W)$, is isomorphic to the Boolean algebra of regular open sets $\mathscr{O}^{\star}\left(p t\left(\operatorname{Id}_{\ll}(W)\right)\right)$ of $\mathscr{O}\left(p t\left(\operatorname{Id}_{\ll}(W)\right)\right)$. This is seen as follows:

STAGE 5. In lattice theoretical terms, the class of regular open sets $\mathscr{O}^{\star}(X)$ of $\mathscr{O}(X)$ is defined as the set of fixpoints of the "double negation" operator $\neg \neg$ of the Heyting algebra $\mathscr{O}(X)$ (cf. Mac Lane and Moerdijk 1991, Ch. I, 8(8)). Let $I \in \operatorname{Id}_{\ll}(W)$ be a Dedekind ideal of $W$. Then the pseudocomplement $\neg I$ of $I$ in $\operatorname{Id}_{\ll}(W)$ is defined by $\neg I:=\left\{z: z \ll y^{\star}\right.$ for all $\left.y \in I\right\}$. As is easily seen, $\neg \neg I=I$ iff $I=\{y: y \ll \sup I\}$ for some $x \in W$. Since $W$ is faithfully embedded by the map $x \mapsto\{y: y \ll x\}$ into $\operatorname{Id}_{\ll}(W)$ the fixpoint set of the double negation is just $W$. Hence, the class of regular open sets $\mathscr{O}^{\star}\left(\operatorname{pt}\left(\operatorname{Id}_{\ll}(W)\right)\right)$ of $\mathscr{O}\left(\mathrm{pt}\left(\operatorname{Id}_{\ll}(W)\right)\right)$ is isomorphic to $W$.

After having completed the stages $1-5$ of the construction process, it is expedient to explicitly state the results obtained sor far in representational terms. Starting with a system $\langle W, \ll\rangle$ such that $\langle W, \ll, \leq\rangle$ is an enriched complete Boolean algebra of regions a topological representation

$$
\mathrm{r}: W \rightarrow \mathscr{O}\left(\operatorname{pt}\left(\operatorname{Id}_{\ll}(W)\right)\right)
$$

has been constructed such that regions are represented by regular open subsets of $\mathrm{pt}\left(\operatorname{Id}_{\ll}(W)\right)$ in a 1-1-fashion. Moreover, the mereological relations of parthood $\leq$ and interior parthood $\ll$ are represented by their topological counterparts:

$$
\begin{aligned}
x \leq y & \Longleftrightarrow \mathrm{r}(x) \subseteq \mathrm{r}(y), \\
x \ll y & \Longleftrightarrow \mathrm{cl}(\mathrm{r}(x)) \subseteq \mathrm{r}(y) \text { and } \operatorname{cl}(\mathrm{r}(x)) \text { compact. }
\end{aligned}
$$


In other words, we have proved a representation theorem for mereological systems of Whiteheadian regions according to which regions are represented in a 1-1-fashion by the regular open sets of a locally compact Hausdorff space. Moreover, the mereological relations such as parthood, interior parthood etc. are represented by their natural topological counterparts. As is easily seen, this representation is essentially unique, i.e., the topological space $\left\langle p t\left(\operatorname{Id}_{\ll}(W)\right), \mathscr{O}\left(\operatorname{pt}\left(\operatorname{Id}_{\ll}(W)\right)\right)\right\rangle$ is uniquely determined up to homeomorphism.

STAGE 6. Let $\mathscr{O}^{\star}(X)$ the complete Boolean algebra of regular open set of a locally compact Hausdorff space $\langle X, \mathscr{O}(X)\rangle$. As is easily shown $\left\langle\mathscr{O}^{\star}(X)\right.$, $\left.\ll_{\mathscr{O}(X)}, \leq\right\rangle$ is an enriched lattice. Then the following concluding result may be proved, which explicitly gives the isomorphism between $\mathscr{O}(X)$ and the Dedekind completion $\operatorname{Id}_{\ll}\left(\mathscr{O}^{\star}(X)\right)$ :

Proposition 4.9. Let $\langle X, \mathscr{O}(X)\rangle$ be a locally compact Hausdorff space. The map $j: \mathscr{O}(X) \rightarrow \operatorname{Id}_{\ll}\left(\mathscr{O}^{\star}(X)\right)$ defined by $j(x):=\left\{y: y \ll_{\mathscr{O}(X)} x \& y \in\right.$ $\left.\mathscr{O}^{\star}(X)\right\}$ is an isomorphism (of continuous Heyting algebras).

Proof. First one has to show that $\mathrm{j}$ is well-defined, i.e., $j(x)$ is indeed a Dedekind ideal of $\mathscr{O}^{\star}(X)$. Obviously, $j(x)$ is downward closed. For $y, y^{\prime} \in$ $j(x)$ one has to show $y \vee y^{\prime} \in j(x)$, i.e., $\operatorname{cl}\left(y \vee y^{\prime}\right)$ is compact and $\operatorname{cl}\left(y \vee y^{\prime}\right) \subseteq x$. In $\mathscr{O}^{\star}(X) y \vee y^{\prime}=\operatorname{int}\left(\operatorname{cl}\left(y \cup y^{\prime}\right)\right)$. Hence $\operatorname{cl}\left(y \vee y^{\prime}\right)=\operatorname{cl}\left(\operatorname{int}\left(\operatorname{cl}\left(y \cup y^{\prime}\right)\right)\right)=$ $\operatorname{cl}(y) \cup \operatorname{cl}\left(y^{\prime}\right) \subseteq x$, since $y, y^{\prime} \ll x$ and $\operatorname{cl}\left(y \vee y^{\prime}\right)$ is compact. Thus, $j(x)$ is an ideal. In order to show that it is a Dedekind ideal one has to show that for $y \in j(x)$ there is a $y^{\prime} \in j(x)$ with $y \ll y^{\prime}$. If $y=x$, taking $y^{\prime}=y$ one obtains $y \ll y^{\prime} \ll x$. Hence, one may assume that $y \neq x$. Since $\mathscr{O}(X)$ is regular, it follows that $\mathscr{O}^{\star}(X)$ satisfies the interpolation property 4.3(iv). Hence we may find a $y^{\prime}$ such that $y \ll y^{\prime} \ll x$. Thus, $j(x)$ is a Dedekind ideal, and $j: \mathscr{O}(X) \rightarrow \operatorname{Id}_{\ll}\left(\mathscr{O}^{\star}(X)\right)$ is well-defined.

Since $\langle X, \mathscr{O}(X)\rangle$ is a regular space, the approximation axiom (A) is known to hold for $\mathscr{O}(X)$. Hence, any open set $x$ is approximated by the class of regular open subsets $y$ which are well below it, i.e., $x=\bigvee\{y: y \in$ $\left.\mathscr{O}^{\star}(X) \& y \ll x\right\}$. This amounts to the fact that $j(x)$ is a monomorphism. More explicitly this may be spelt out as follows: consider the map sup : $\operatorname{Id}_{\ll}\left(\mathscr{O}^{\star}(X)\right) \rightarrow \mathscr{O}(X)$, defined by $I \mapsto \sup (I)$. Then we get $\sup (j(x))=x$. Hence $j$ must be a monomorphism.

Next one proves that $j$ is also an epimorphism. Let $I \in \operatorname{Id}_{\ll}\left(\mathscr{O}^{\star}(X)\right)$ and $x=\sup I$. Define $\downarrow I:=\{y: y \leq x$ for some $x \in I, y \in \mathscr{O}(X)\}$. Then 
$x=\sup \downarrow I$ and, since $\mathscr{O}(X)$ is a continuous lattice, one has $\downarrow \downarrow x=\{y$ : $y \ll x, y \in \mathscr{O}(X)\} \subseteq \downarrow I$ by (Gierz et al. 1980, I-(2.1)(2), p. 57). Hence $\downarrow \downarrow x \cap \mathscr{O}^{\star}(X) \subseteq I$.

The reverse implication is proved as follows. Let $y \in I$. Since $I$ is a Dedekind ideal there is a $z \in I$ such that $y \ll z \leq x$. Hence $y \ll x$, i.e., $I \subseteq \downarrow \downarrow x \cap \mathscr{O}^{\star}(X)$. Thus every Dedekind ideal is of the form $j(x)=\downarrow \downarrow$ $x \cap \mathscr{O}^{\star}(X)$, i.e., $j$ is an epimorphism. As one has already proved that $j$ is a monomorphism it follows that $j$ is an isomorphism. Obviously, $x \leq y$ iff $j(x) \leq j(y)$ and hence $x \ll y$ iff $j(x) \ll j(y)$. Hence, $\mathscr{O}(X)$ and $\operatorname{Id}_{\ll}\left(\mathscr{O}^{\star}(X)\right)$ are isomorphic as continuous Heyting algebras.

In category theoretical terms $(*)$ and Proposition 4.9 may be succinctly expressed as follows: denote the category of enriched complete Boolean algebras by $\mathbf{B}(+)$ and the category of continuous Heyting algebras by $\mathbf{C H}$. Then we may regard $\mathrm{Id}_{\ll}$ as a functor $\mathrm{Id}_{\ll}: \mathbf{B}(+) \rightarrow \mathbf{C H}$. A functor $\mathscr{O}^{\star}: \mathbf{C H} \rightarrow$ $\mathbf{B}\left({ }^{\star}\right)$ may be defined by mapping a continuous Heyting algebra $H$ onto the enriched complete Boolean algebra $\left\langle\mathscr{O}^{\star}(H), \leq, \ll_{H}\right\rangle$, where $\mathscr{O}^{\star}(H)$ is the fixpoint of the double negation operator $\neg \neg H$ which is in topological terms just the Boolean algebra of regular open sets. Then $\mathrm{Id}_{\ll}$ and $\mathscr{O}^{\star}$ are inverse to each other. In other words, the categories $\mathbf{B}(+)$ and $\mathbf{C H}$ are equivalent.

\section{Concluding remarks}

Since Process and Reality (1929) in which Whitehead gave an informal (and apparently inconsistent) sketch of a what later was to be called the Whiteheadian account of space quite a few authors have attempted to render precise Whitehead's informal approach (cf. Biacino and Gerla 1991 and 1996, Clarke 1981 and 1985, Forrest 1996, Gerla and Tortora 1992, Grzegorczyk 1960, Roeper 1997). Independently from Whitehead, the mathematical theory of pointless topology may be considered as an immense generalization of the Whiteheadian programme. As the starting point of this mathematical theory one may take Stone's representation theorem of 1936. An authoritative modern account may be found in Johnstone 1981. These developments have taken place inside mathematics and have largely gone unnoticed by philosophers. This is evidenced by the fact that no philosopher working on the Whiteheadian theory of space has ever mentioned Stone's path-breaking work of 1936, to say nothing of other more modern mathematical sources. Obviously, this paper is not the appropriate place to comment on the huge 
and complex mathematical developments of pointless topology and lattice theory. ${ }^{6}$ Rather, I'd like to be content of making some remarks on some of the more recent attempts of philosophers to render precise Whitehead's informal proposals. In particular, I'd like to deal with Roeper's lengthy article of 1997 (Roeper 1997). In this paper Roeper has obtained a similar result as Proposition 4.5 and $(*)$. His basic concept is the notion of a region-based topology $\mathscr{R}$ defined as a structure $\mathscr{R}=\langle\Omega, \infty, \Delta\rangle$, where $\Omega$ is a Boolean algebra of regions, $\infty$ the 2-place predicate of connection, and $\Delta$ the set of limited regions, the latter two being subject to ten axioms, roughly corresponding to our group of Assumptions II. Then Roeper's Main Theorem (Roeper 1997, 279) reads as follows:

Roeper's Theorem. The complete region-based topologies correspond 1-1 (modulo isomorphisms and homeomorphisms respectively) to the locally compact Hausdorff spaces.

In contrast to the approach presented in this paper, Roeper employs the non-mereological notions of connection $\infty$ and limitedness $(\Delta)$ of regions. Hence, for mereological purists, his result may leave something to desire. Somewhat surprisingly, Roeper does not use the available results of "pointless topology" (cf. Johnstone 1981, Mac Lane, Moerdijk 1991). Consequently, he has to prove his results almost from scratch. Nevertheless, his account and that of the present paper show important similarities. Hence, one may ask how Roeper's apparently non-mereological concepts of connection and limitedness could have been avoided in the approach developed in this paper. The general answer is that the notion of compactness may be used to define appropriate substitutes for the non-mereological concepts of limitedness and connection. I cannot go into the details of Roeper's approach which is after all, rather involved, just a few remarks. In the bulk of his paper he treats limitedness as primitive. Later, however, he defines limitedness, at least for some region-based topologies, in terms of the connection and Boolean operations (cf. Roeper 1997, section 7). Rather, I'd like to invoke an example which is paradigmatic for the Whiteheadian account of space, to wit, Euclidean space $\mathbb{R}^{n}$. According to the well-known theorem of Borel-Lebesgue a subset $A \subseteq \mathbb{R}^{n}$ is compact iff $A$ is closed and bounded. Hence, one may topologically define a set $A$ as bounded iff its closure $\operatorname{cl}(A)$ is compact. In this way, compactness may be considered as the topological substitute

\footnotetext{
${ }^{6}$ Some historical remarks on Stone's theorem may be found in Piazza 1995.
} 
for limitedness. Hence, limitedness may not be considered as an absolutely primitive notion of Roeper's approach, and we are left with two primitive notions, namely, connection and the Boolean structure $\Omega$ which may be regarded as being encapsulated in the parthood relation $\leq$. Now Roeper could have defined (actually he does not) the connection relation $\infty$ by a notion of interior parthood $\ll \mathscr{R}$ as follows (cf. also Biancino and Gerla 1996):

$$
x \infty y \stackrel{\mathrm{df}}{\Longleftrightarrow} x \ll \mathscr{R} y^{\star}, \quad y^{\star} \text { the Boolean complement of } y .
$$

In topological terms $\ll \mathscr{R}$ turns out to be defined as follows: $x \ll \mathscr{R} y$ iff $\operatorname{cl}(x) \subseteq y$. For compact spaces, $\ll \mathscr{R}$ coincides with the relation of interior parthood $\ll$, i.e., invoking the theory of continuous lattices it could have been defined in terms of $\leq$. Hence, the basic conceptual vocabularies of Roeper's account and that of the present paper are essentially equivalent. Thus it should not be too surprising that the two accounts come to similar results. We may conclude that in Roeper's account the non-mereological concepts of connection and limitedness only play superficial roles and may be eliminated in favour of a purely mereological approach. Several authors have denied that such a purely mereological account is possible. I hope to have defeated this claim in this paper. Moreover, I hope to have rendered evident the fact that lattice theory and modern accounts of pointless topology offer a lot of ready-made tools and devices which may be employed in the realms of mereology, in particular, Whiteheadian theory of space.

\section{References}

Banaschewsky, B, Hofmann, R.-E., 1981, Continuous Lattices (eds.), Lecture Notes in Mathematics 871, Berlin, Heidelberg, New York, Springer.

Biacino, L., Gerla, G., 1991, "Connection Structures", Notre Dame Journal of Formal Logic 32, 242-247.

Biacino, L., Gerla, G., 1996, "Connection structures: Grzegorczyk's and Whitehead's definitions of point", Notre Dame Journal of Formal Logic 37, 431-439.

Clarke, B. L., 1981, "A calculus of individuals based on 'connection' ", Notre Dame Journal of Formal Logic 22, 204-218.

Clarke, B. L., 1985, "Individuals and points", Notre Dame Journal of Formal Logic 26, 61-75.

Davey, B. A., Priestley, H. A., 1990, Introduction to Lattices and Order, Cambridge, Cambridge University Press. 
Forrest, P., 1996, "How innocent is mereology?", Analysis 56, 127-131.

Gerla, G., Tortora, R., 1992, "La relazione di connessione in A. N. Whitehead: Aspetti matematici", Epistemologia 15, 351-364.

Gierz, G., Hofmann, K. H., Keimel, K., Lawson, J. D., Mislove, M., Scott, D. S., 1980, A Compendium of Continuous Lattices, Berlin, Heidelberg, New York, Springer.

Gierz, G., Keimel, K., 1981, "Continuous ideal completions and compactifications", in B. Banaschewsky and R.-E. Hofmann (eds.), 97-124.

Grzegorczyk, A., 1960, "Axiomatizability of geometry without points", Synthese 12, $228-235$.

Johnstone, P. T., 1982, Stone Spaces, Cambridge, Cambridge University Press.

Mac Lane, S., Moerdijk, I., 1992, Sheaves in Geometry and Logic. A First Introduction to Topos Theory, New York, Springer.

Mormann, T., 1998, "Neither mereology nor Whiteheadian account of space yet convicted" (to appear in Analysis).

Mormann 1998a, "Topological representations of mereological systems" (to appear in Poznan Studies in the Philosophy of the Sciences and the Humanities, Amsterdam, Rodopi).

Piazza, M., 1995, “'One must always topologize’: Il teorema di Stone, la ‘topologia influente' e l'epistemologia matematica", Rivista di storia della scienza (ser. II), $3,1-24$.

Roeper, P., 1997, "Region-based topology", Journal of Philosophical Logic 26, 251-309.

Stone, M., 1936, "The theory of representations for Boolean algebras", Transactions of the American Mathematical Society 40, 37-111.

Vickers, S., 1989, Topology via Logic, Cambridge, Cambridge University Press.

Whitehead, A. N., 1929, Process and Reality. An Essay in Cosmology, New York, Macmillan.

Thomas Mormann

IPLW, Universität München

Ludwigstr. 31

D-80539 München, Germany 